\title{
Prevalence of glaucoma in patients with moderate to severe obstructive sleep apnea: ocular morbidity and outcomes in a 3 year follow-up study
}

SP Hashim ${ }^{1}$, FA Al Mansouri', M Farouk', AA Al Hashemi ${ }^{2}$ and R Singh ${ }^{3}$
${ }^{1}$ Ophthalmology Section, Hamad Medical

Corporation, Doha, Qatar

2Pulmonary Division, Department of Medicine, Hamad Medical Corporation, Doha, Qatar

${ }^{3}$ Biostatitics Division, Department of Cardiology, Hamad Medical Corporation, Doha, Qatar

Correspondence: SP Hashim, Ophthalmology Section, Hamad Medical Corporation, PO Box 3050, Doha, Qatar

Tel: +974 55 637841;

Fax: +974 44393239

E-mail: drshakeel32@

hotmail.com

Received: 14 November 2013

Accepted in revised form:

8 July 2014

Published online:

15 August 2014
This article has been corrected since Advance Online Publication and an erratum is also printed in this issue
Abstract
Purpose This study was conducted to investigate the prevalence and progression of glaucoma in patients receiving treatment for obstructive sleep apnea (OSA). We also investigated whether there is an association between severity of OSA and the incidence of glaucoma.
Methods A total of 39 patients aged $>30$ years who had been diagnosed with moderate and severe OSA in the sleep clinic at Hamad General Hospital were assessed for the presence of glaucoma. The severity of OSA was graded as mild, moderate, or severe based on American Association of Sleep Medicine (AASM) criteria using the apnea hypopnea index. Before enrollment, all patients underwent a complete ophthalmic examination including serial visual field tests, optical coherence tomography (OCT) with fundus photographs, and pachymetry. Enrolled patients were followed up in the ophthalmology outpatient clinic and sleep clinic for a period of 3 years.
Results Examinations found that 8 (20.5\%; 95\% confidence interval (CI) 9.9-37\%) of the 39 patients with OSA had glaucoma. Six (75\%; 95\% CI 36-96\%) of these patients had normal-tension glaucoma (NTG) and two (25\%; 95\% CI 4.5-64.4\%) patients had high- tension glaucoma. Among the 27 patients with severe OSA, 7 (25.9\%; 95\% CI 8-34\%) had glaucoma, and among 12 patients with moderate OSA, $1(8.3 \%$; 95\% CI $0.1-15 \%)$ had

glaucoma. During the course of follow-up, two patients who previously did not have glaucoma were reclassified as NTG and two patients with glaucoma deteriorated. A higher prevalence of glaucoma in the severe OSA group compared with the moderate OSA group was found, albeit a statistically significant difference could not be attained $(P=0.4)$.

Conclusions Our study showed that severe OSA is an important risk factor for developing glaucoma. Adequate treatment of OSA, along with optimal ophthalmic care, resulted in better control of glaucoma. Eye (2014) 28, 1304-1309; doi:10.1038/eye.2014.195; published online 15 August 2014

Introduction

Sleep apnea is a common disorder characterized by brief interruptions of breathing during sleep, and sleep apnea syndrome is defined as episodes of apnea and hypopnea during sleep accompanied by symptoms of functional impairment after sleep. ${ }^{1}$

Obstructive sleep apnea (OSA) is a type of sleep apnea that results from complete or partial collapse of the pharyngeal airway during sleep, and is characterized by brief interruptions of breathing.

OSA increases the risk for cardiovascular morbidity and mortality, and can also cause hypertension and increased insulin resistance. OSA has also been associated with daytime 
sleepiness, which can lead to automobile accidents.

Oxygen levels in tissues decrease significantly during sleep in patients with OSA, resulting in changes in brain metabolism and brain activation response. ${ }^{2}$ Glaucoma is the second leading cause of blindness worldwide, and in the year 2020, an estimated 79.6 million people will be affected by the disease. ${ }^{3}$ Glaucoma is defined as a chronic progressive optic neuropathy, ${ }^{4}$ and it is usually asymptomatic until the disease is well advanced. Even in developed countries, only $\sim 50 \%$ of probable glaucoma patients are officially diagnosed with the disease, and the rate of diagnosis in developing countries is probably much lower. ${ }^{3}$ Prevalence of OSA in the general population showed wide variation, affecting men more than women. Values ranging from 3 to $7 \%$ were mentioned in various studies, and found to be higher in the studies from Asia. ${ }^{5}$ Previous studies have shown an association between OSA and glaucoma in general and NTG in particular. ${ }^{6-13}$ This study was conducted to investigate the prevalence and progression of glaucoma in patients receiving treatment for OSA. We also investigated whether there is an association between the severity of OSA and the incidence of glaucoma.

\section{Patients and methods}

A total of 39 consecutive patients (34 males and 5 females) aged $>30$ years, and who had been diagnosed with moderate to severe OSA at the sleep clinic at Hamad General Hospital were assessed for the presence of glaucoma. The severity of OSA was graded using a diagnostic portable sleep tool (Stardust II sleep recorder, Philips-Respironics, Philips Healthcare, Best, The Netherlands) and classified as mild, moderate, or severe according to the apnea hypopnea index (AHI).

The studies were manually scored by a trained staff based on the American Academy of Sleep Medicine (AASM) guidelines. Apnea is defined as $<10 \%$ of the base line airflow lasting $10 \mathrm{~s}$ or more, and hypopnea is $<70 \%$ of airflow or effort lasting $10 \mathrm{~s}$ or more with at least $4 \%$ oxyhemoglobin desaturation. ${ }^{14}$

Accordingly, 5-15 episodes of apnea or hypopnea $/ \mathrm{h}$ is classified as a mild disorder, $15-30$ episodes $/ \mathrm{h}$ is a moderate disorder, and $>30$ episodes $/ \mathrm{h}$ is classified as severe obstructive sleep apnea. ${ }^{15}$

All patients enrolled in this study underwent a complete ophthalmic examination, serial visual field tests, optical coherence tomography (OCT) with fundus photographs and pachymetry. Patients were followed up in the ophthalmology outpatient clinic and the sleep clinic for a period of 3 years, and were especially monitored for the presence and progression of glaucoma and the control of OSA. The visual fields using 24-2 Swedish Interactive Threshold Algorithm standard program was done in all patients with Humphrey field analyzer II (Carl Zeiss Meditec, Inc., Dublin, CA, USA). Using spectral domain OCT (Carl Zeiss Meditec, Inc.), RNFL (retinal nerve fiber layer), and ONH (optic nerve head): optic disc cube $200 \times 200$ program was performed to find out the average RNFL thickness and RNFL symmetry. The visual field and OCT results were interpreted by glaucoma consultants (SPH, FAAM) with the help of ophthalmic diagnostic consultant (MF). Special attention was made on the RNFL quadrants and clock hour maps. These findings were compared with optic disc changes in the form of diffuse or localized neuroretinal rim thinning, asymmetric cup disc ratio, splinter- or flame-shaped hemorrhages on the optic disc, nerve fiber defects using red-free green light and peripapillary atrophic changes in the beta-zone. Glaucoma was diagnosed on the basis of optic disc abnormalities accompanied by corresponding changes in visual fields and OCT results. Anderson criteria for early glaucomatous visual field changes were used to diagnose glaucoma. (1) Presence of a cluster of three or more non-edge points on the pattern deviation plot, deviating at a $P<5 \%$, and one of them reduced to $P<1 \%$. (2) Pattern standard deviation (PSD) with a value $P<5 \%$. (3) Glaucoma hemi-field test outside normal limits. ${ }^{16}$

Presence of any two of the above three criteria along with corresponding changes in the optic disc and the OCT-NFL were considered for the diagnosis of glaucoma. Or those patients who did not meet the norms to diagnose glaucoma initially, but later develop any two of the abovementioned visual field criteria with a progressive reduction in OCT-NFL thickness accompanied by optic disc changes including flame-shaped hemorrhages and neuroretinal rim thinning in the follow-up visits were also included in the glaucoma category.

All patients were reviewed every 6 months for a period of 3 years. During each visit, the visual fields and OCTs were repeated. Overview of the visual fields and the OCT glaucoma progression analysis were done to track the status of each patient with or without glaucoma. Accordingly, development of new scotomas or increase in the depth of scotoma in the same three or more test point locations in the pattern deviation plot in repeated visual field tests compared with the baseline visual field was considered as glaucoma progression, in the presence of matching changes in the optic disc or OCT. A consistently documented reduction of NFL thickness by repeated OCT-GPA analysis with corresponding changes in the optic disc, with or without any visual field changes, was also considered as a sign of progression of glaucoma.

Once the glaucoma was diagnosed, patients with a corrected IOP $<21 \mathrm{~mm} \mathrm{Hg}$ at all visits were classified as normal tension glaucoma (NTG), and those with a 
corrected IOP $\geq 21 \mathrm{~mm} \mathrm{Hg}$ were classified as high tension glaucoma (HTG).

Descriptive statistics was performed in the form of mean and standard deviation for interval variables. Frequency and percentages for categorical variables were calculated with $95 \%$ confidence interval (CI) using continuity correction factor. Student's t-tests (unpaired) were used to see significant difference between the categories severe and moderate OSA for interval variables like age, $\mathrm{AHI} / \mathrm{h}$, visual acuity, IOP (mmHg), CCT (microns), VCDR, RNFL (microns), MD and PSD. P-value of 0.05 was considered as statistically significant level. SPSS 21.0 statistical package (SPSS Inc., Chicago, IL, USA) was used for the analysis.

This study was approved by the Institutional Review Board of the Medical Research Centre, Hamad Medical Corporation. Written informed consent was obtained from all the enrolled patients.

\section{Results}

Our study population included patients of Asian, African, and native Arab descent. The majority of patients $(87.17 \%)$ were male, and ages ranged from 31 to 74 years, with a mean age of $52.03 \pm 11.17$ years. In this study, $27(69.2 \%)$ patients had severe OSA and $12(30.8 \%)$ had moderate OSA. Mean duration of the study period was $2.9 \pm 0.63$ years.

Table 1 Comparison of baseline characteristics according to severity of OSA

\begin{tabular}{lccc}
\hline Variable & Severe OSA & Moderate OSA & P-value \\
\hline Age (years) & $51.89 \pm 11.62$ & $52.33 \pm 10.57$ & 0.90 \\
AHI (/h) & $39.89 \pm 8.8$ & $24.36 \pm 6.01$ & 0.001 \\
Visual acuity & $0.93 \pm 0.10$ & $0.94 \pm 0.21$ & 0.97 \\
IOP (mm Hg) & $17.0 \pm 3.4$ & $14.5 \pm 2.0$ & 0.02 \\
CCT (microns) & $536.04 \pm 31.0$ & $541.81 \pm 37.1$ & 0.62 \\
VCDR & $0.53 \pm 0.12$ & $0.47 \pm 0.15$ & 0.25 \\
RNFL (microns) & $86.6 \pm 6.6$ & $86.81 \pm 3.9$ & 0.39 \\
MD & $-1.87 \pm 2.1$ & $-1.87 \pm 2.3$ & 0.99 \\
PSD & $2.12 \pm 1.19$ & $2.06 \pm 1.32$ & 0.38 \\
\hline
\end{tabular}

Values denote mean \pm SD.
Of the 39 patients with OSA, $8(20.51 \%$; $95 \%$ CI 9.9$37 \%)$ patients had glaucoma; $6(75 \% ; 95 \%$ CI 36-96\%) of these patients had NTG, and 2 (25\%; 95\% CI 4.5-64.4\%) patients had HTG. Among the 27 patients with severe OSA, 7 (25.92\%; 95\% CI 8-34\%) had glaucoma. Among the 12 patients with moderate OSA, 1 (8.33\%; 95\% CI 0.1-15\%) had glaucoma. (Details of other characteristics are given in Tables 1 and 2.)

We found that $87.5 \%$ of the glaucoma patients in our study was from severe OSA group and $12.5 \%$ was from moderate OSA group (Figure 1).

Nevertheless, a statistically significant difference in the prevalence of glaucoma between the two groups could not be attained $(P=0.4)$. Three patients with glaucoma and one patient without glaucoma had a positive family history of glaucoma. One patient showed signs of pseudo-exfoliation, but not glaucoma. Gonioscopy revealed open angle and normal pigmentation of trabecular meshwork in all patients. There were two patients with high myopia, but only one had NTG. Seven out of the eight glaucoma patients were diagnosed to have glaucoma for the first time after their enrollment in the study. In all, 11 patients had both diabetes mellitus and hypertension, 9 had diabetes mellitus alone, and 7 had only hypertension; however, no retinopathy was detected in any of these patients. Three patients had cataracts, and one of them underwent bilateral cataract surgery and the other underwent unilateral cataract surgery. All patients diagnosed with glaucoma were treated with latanoprost $0.005 \%$ eye drops at bed time. Also, one patient with HTG received Cosopt (timolol $0.5 \%$ and trusopt $2 \%$ fixed combination) twice daily (b.i.d.), and another patient who showed advanced glaucomatous changes was prescribed Cosopt eye drops (b.i.d.) and brimonidine $0.15 \%$ eye drops (b.i.d.).

Following a detailed evaluation in the sleep lab, all patients were advised by the physician to begin treatment with continuous positive airway pressure (CPAP). Compliance to the prescribed CPAP treatment varied between 40 and $50 \%$ during the study period. Three patients underwent surgery to correct OSA. Two patients with severe OSA discontinued CPAP treatment

Table 2 Baseline characteristics of glaucoma and non-glaucoma groups

\begin{tabular}{lcccc}
\hline Variable & Glaucoma right eye & Glaucoma left eye & Non-glaucoma right eye & Non-glaucoma left eye \\
\hline Visual acuity & $0.9 \pm 0.2$ & $0.9 \pm 0.1$ & $0.9 \pm 0.2$ & $0.9 \pm 0.2$ \\
IOP (mm Hg) & $17.29 \pm 2.7$ & $19 \pm 5.5$ & $15.97 \pm 3.1$ & $15.71 \pm 3.2$ \\
CCT (microns) & $536.71 \pm 20.7$ & $542.75 \pm 25.57$ & $539.16 \pm 36$ & $537.61 \pm 35.4$ \\
VCDR & $0.66 \pm 0.1$ & $0.61 \pm 0.15$ & $0.49 \pm 1.2$ & $0.48 \pm 1.1$ \\
RNFL (microns) & $79.86 \pm 7$ & $79 \pm 6.3$ & $88.1 \pm 4.9$ & $88.48 \pm 5.5$ \\
MD & $-3.18 \pm 2.5$ & $-5.09 \pm 4.4$ & $-1.18 \pm 1.64$ & $-1.4 \pm 1.57$ \\
PSD & $3.49 \pm 1.87$ & $3.61 \pm 2.91$ & $1.73 \pm 0.46$ & $1.7 \pm 0.5$ \\
\hline
\end{tabular}

Values denote mean \pm SD. 
when they achieved satisfactory results in the sleep study following reduction in body weight. One of these patients attained weight loss following bariatric surgery. One patient with severe OSA developed myocardial infarction and underwent angioplasty. Three patients (7.7\%) with severe OSA with glaucoma and good control of OSA remained stable all through. During the course of follow-up, two $(5.1 \%)$ patients with poorly controlled OSA, who previously did not have glaucoma, were reclassified as NTG when they developed changes according to the specified criteria to diagnose glaucoma.

Besides, as per the criteria to detect progression of glaucoma, two patients with poor control of OSA deteriorated during the follow-up (Figure 2).

\section{Discussion}

The main cause of all complications associated with OSA is the decreased oxygenation of vital structures during sleep.

Hyperventilation and resultant hypoxia occur normally during sleep, and particularly during rapid eye movement (REM) sleep. ${ }^{17}$ During the deepest stages of

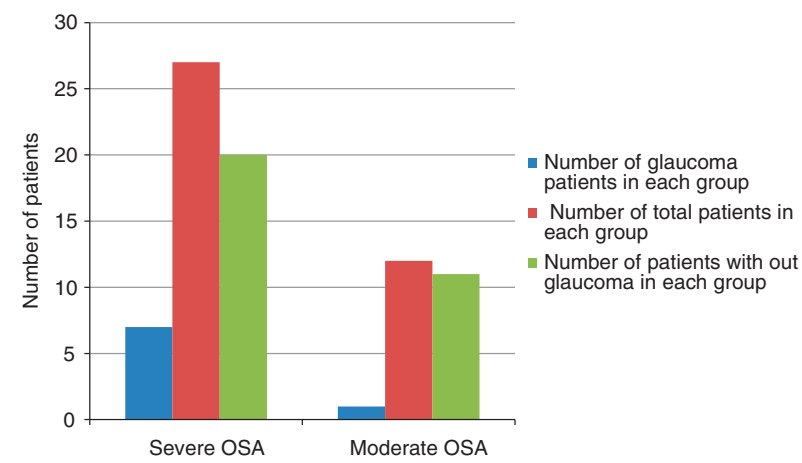

Figure1 Prevalence of glaucoma in severe and moderate OSA. sleep, autoregulation of cerebral blood flow does not function perfectly, even in normal subjects, and this regulation appears to be severely disturbed in OSA. ${ }^{18}$ In patients with OSA, blood flow to the ONH may be altered and may result in glaucoma. ${ }^{9}$

The ophthalmic artery is a branch of the internal carotid artery and shows similar characteristics regarding autoregulation of blood flow. In patients with OSA, the cerebral blood flow in response to hypoxia returns to normal levels after treatment with CPAP for a period of 6 weeks. ${ }^{18}$

A high prevalence of glaucoma was found in patients with OSA, and especially in severe cases. ${ }^{19}$ The prevalence of glaucoma in OSA shows a wide range of fluctuation. Bendel et $a l^{20}$ reported a higher prevalence of NTG (27\%) in patients with OSA. According to another study, the prevalence of NTG in all types of OSA was $5.7 \%$, and increased to $7.1 \%$ in moderate and severe OSA cases. ${ }^{7}$ Tsang et $a l^{21}$ reported that moderate to severe OSA was associated with a higher incidence of visual field defects and glaucomatous changes in the optic nerve. These findings are in accordance with our study results. Although we found a higher prevalence of glaucoma in the severe OSA group compared with the moderate OSA group, a statistically significant difference could not be attained $(P=0.4)$. This was primarily due to the low number of study subjects. We also found that, in the category of poorly controlled severe OSA, a deterioration of the visual field and OCT ensued in four patients. Two of these were already classified as glaucoma and the other two were from the previously non-glaucoma group, and the latter was eventually included in the glaucoma category.

Sergi et al ${ }^{11}$ reported that the prevalence of NTG was higher in OSA patients compared with a control Caucasian population of same age, and that OSA may be an important risk factor for NTG. These studies further

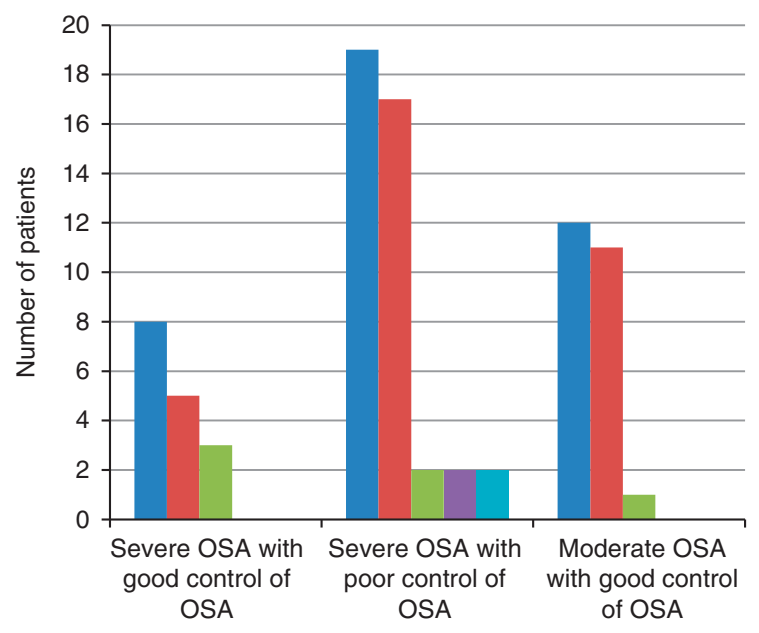

Total patients in the each group

- Number of non- glaucoma patients on initial check up

- Number of glaucoma patients on initial check up

- Number of non- glaucoma patients developed glaucoma during follow up

- Number of glaucoma patients deteriorated during follow up

Figure 2 Control of OSA and glaucoma. 
support our findings that OSA is an important risk factor for the development and progression of glaucoma, and especially NTG.

Mojon et $a l^{22}$ reported that sleep apnea was more prevalent among patients with POAG than a normal control group. In a 5-year retrospective study published in 2013, Lin et $a l^{23}$ found a statistically significant increase in the incidence of open angle glaucoma in OSA patients compared with non-OSA patients.

Silva et al ${ }^{10}$ reported that in a $24-\mathrm{h}$ IOP monitoring study involving 11 patients with OSA, the mean IOP was highest at 0600 hours and while the patients were in the lying position. Although the study is not specifically mentioning whether it is supine or prone position, the increase in IOP during lying can be a critical factor in the development or progression of glaucoma in patients with OSA.

On the contrary, a study conducted by Girkin et al ${ }^{24}$ failed to support the relevance of sleep apnea in comparison to other risk factors in the eventual development of glaucoma. However, the significance of these findings is in doubt, because there is overwhelming evidence supporting OSA as a risk factor for the development of glaucoma.

Boland et $a l^{13}$ recently conducted a detailed evaluation of factors that can contribute to the development of glaucoma, and sleep apnea was considered to be one of the risk factors. Vascular insufficiency and elevated IOP can cause death of retinal ganglion cells and lead to development of glaucoma. Vascular insufficiency may result from reduced ocular blood flow, decreased ocular perfusion pressure, generalized vascular deregulation, or increased trans-lamina cribrosa pressure. ${ }^{25}$

One significant drawback of the current study is the relatively small number of patients studied. However, the number of subjects was kept small to ensure meticulous follow-up for a period of 3 years and avoid errors in assessments of visual fields and OCT results. Patients with doubtful findings were screened repeatedly during their follow-up visits to obtain conclusive evidence for classifying them as glaucoma or non-glaucoma subjects.

Although control of IOP is the most important aspect of managing NTG, it is imperative to explore the presence of other factors that may influence perfusion of the $\mathrm{ONH} .{ }^{26}$ Kremmer et $a l^{8}$ reported that two cases of NTG became stabilized after treatment of OSA with nasal CPAP, and this approach may be applicable for managing other types of glaucoma with symptoms of OSA. Considering the findings described above, and our own results, we strongly endorse the need to screen all OSA patients for glaucoma. Also, glaucoma patients with symptoms suggestive of OSA should be advised to undergo a detailed sleep study; a concept also supported by Marcus et al. ${ }^{12}$

\section{Conclusions}

Our study showed that severe OSA is an important risk factor for developing glaucoma. In addition, better control of glaucoma can be achieved by adequate treatment of OSA by careful titration of CPAP treatment along with optimal ophthalmic care.

This study highlights the need to increase awareness among both general practice physicians and ophthalmologists of the association between glaucoma and OSA.

\section{Summary}

What was known before
Normal-tension glaucoma associated with obstructive
sleep apnea (OSA) and high-tension glaucoma also shows
a higher prevalence in patients with OSA.
What this study adds
Confirms the association of glaucoma and sleep apnea in
a prospective study, over a period of 3 years. Patients with
OSA and without glaucoma can develop glaucoma if OSA
is poorly controlled. Strict control of OSA can contribute
to the better management of glaucoma and stabilization of
the visual fields.

\section{Conflict of interest}

The authors declare no conflict of interest.

\section{Acknowledgements}

The authors acknowledge the support of the Medical Research Centre, Hamad Medical Corporation, Doha, Qatar.

\section{References}

1 Young T, Palta M, Dempsey J, Skatrud J, Weber S, Badr S. The occurrence of sleep disordered breathing among middle aged adults. N Engl J Med 1993; 328: 1230-1235.

2 Ayalon L, Peterson S. Functional central nervous system imaging in the investigation of obstructive sleep apnoea. Curr Opin Pulm Med 2007; 13(6): 479-483.

3 Quigley HA, Broman AT. The number of people with glaucoma worldwide in 2010 and 2020. Br J Ophthalmol 2006; 90(3): 262-267.

4 Foster PJ, Buhrmann R, Quigley HA, Johnson GJ. The definition of glaucoma in prevalence surveys. Br J Ophthalmol 2002; 86(2): 238-242.

5 Punjabi NM. The epidemiology of adult obstructive sleep apnea. Proc Am Thorac Soc 2008; 5: 136-143. 
6 Walsh JT, Montplaisir J. Familial glaucoma with sleep apnea: a new syndrome? Thorax 1982; 37(11): 845-849

7 Lin PW, Friedman M, Lin HC, Chang HW, Wilson M, Lin MC. Normal tension glaucoma in patients with OSA/ hypopnea syndrome. J Glaucoma 2011; 20(9): 553-558.

8 Kremmer S, Niederdräing N, Ayertey HD, Steuhl KP, Selbach JM. Obstructive sleep apnea syndrome, normal tension glaucoma and nCPAP therapy- a short note. Sleep 2003; 26(2): 161-162.

9 Faridi O, Park SC, Liebmann JM, Ritch R. Glaucoma and obstructive sleep apnea syndrome. Clin Experiment Ophthalmol 2012; 40(4): 408-419.

10 Silva M, Weber S. Study on 24 hours IOP study and glaucoma frequency in patients with obstructive sleep apnea syndrome. Rev Bras Oftalmol 2009; 68(6): 338-343.

11 Sergi M, Salerno DE, Rizzi M, Blini M, Andreoli A, Messenio $\mathrm{D}$ et al. Prevalence of normal tension glaucoma in obstructive sleep apnea syndrome patients. J Glaucoma 2007; 16(1): 42-46.

12 Marcus DM, Costarides AP, Gokhale P, Papastergiou G, Miller JJ, Johnson $\mathrm{MH}$ et al. Sleep disorders: a risk factor for normal-tension glaucoma? J Glaucoma 2001; 10(3): 177-183.

13 Boland MV, Quigley HA. Risk factors and open-angle glaucoma: classification and application. J Glaucoma 2007; 16(4): 406-418.

14 Iber C, Ancoli-Israel S, Chesson A, Quan SF. for the American Academy of Sleep Medicine. The AASM Manual for Scoring of Sleep and Associated Events: Rules, Terminology and Technical Specifications. 1st edn. American Academy of Sleep Medicine: Westchester, IL, USA, 2007.

15 American Academy of Sleepmedicine task force. Sleep related breathing disorders in adults: recommendations for syndrome definition and measurement techniques in clinical research; the report of an American academy of sleep medicine task force. Sleep 1999; 22(5): 667-689.
16 Anderson DR, Patella VM. Interpretation of a single visual field. In: Automated Static Perimetry. 2nd edn. Mosby:

St. Louis, MO, USA, 1999, pp 121-190.

17 Douglas NJ, White DP, Pickett CK, Weil JV, Zwillich CW. Respiration during sleep in normal man. Thorax 1982; 37: 840-844.

18 Foster GE, Hanly PJ, Ostrowski M, Poulin MJ. Effects of continuous positive air pressure on cerebral vascular response to hypoxia in patients with OSA. Am J Respir Crit Care Med 2007; 175: 720-725.

19 Karakucuk S, Goktas S, Aksu M, Erdogan N, Demirci S, Oner A et al. Ocular blood flow in obstructive sleep apnea syndrome (OSAS). Graefes Arch Clin Exp Ophthalmol 2008; 246(1): 129-134.

20 Bendel RE, Kaplan J, Heckman M, Fredrickson PA, Lin SC. Prevalence of glaucoma in patients with OSA,- a cross sectional case series. Eye (Lond) 2008; 22(9): 1105-1191.

21 Tsang CS, Chong SL, Ho CK, Li MF. Moderate to severe obstructive sleep apnea patients is associated with higher incidence of visual field defects. Eye (Lond) 2006; 20(1): 38-42.

22 Mojon DS, Hess CW, Goldblum D, Bohnke M, Korner F, Mathis J. Primary open angle glaucoma is associated with sleep apnea syndrome. Ophthalmologica 2000; 214(2): $115-118$.

23 Lin CC, Hu CC, Ho JD, Chiu HW, Lin HC. Obstructive sleep apnea and risk of glaucoma a population based matched cohort study. Ophthalmology 2013; 120(8): 1559-1564.

24 Girkin CA, McGwin Jr, G, McNeal SF, Owsley C. Is there an association between pre-existing sleep apnea and the development of glaucoma? Br J Ophthalmol 2006; 90: 679-681

25 Agarwal R, Gupta SK, Agarwal P, Saxena R, Agrawal SS. Current concepts in the pathophysiology of glaucoma. Indian J Ophthalmol 2009; 57(4): 257-266.

26 Shields MB. Normal-tension glaucoma: is it different from primary open-angle glaucoma? Curr Opin Ophthalmol 2008; 19(2): $85-88$. 